\title{
Dynamic coupled thermoelastic response of thin spherical shells
}

\author{
M. Amiri ${ }^{1}$, M. Bateni ${ }^{2}$, M.R. Eslami ${ }^{2 *}$ \\ ${ }^{1}$ Islamic Azad University, Mechanical Engineering Department, Tehran, Iran \\ ${ }^{2}$ Amirkabir University of Technology, Mechanical Engineering Department, Tehran, Iran
}

\begin{abstract}
This work presents the dynamic coupled thermoelastic response of thin spherical shells subjected to a transverse thermal shock. The shell under investigation is assumed to be sufficiently thin to neglect the curvature effect through the thickness. Also, the shell is considered to be constituted from homogenous and isotropic material. The Hooke and Fourier laws, respectively, are considered to govern the mechanical and thermal behavior of the constituent material. Under the applied load, it is assumed that the displacements of the shell remain in the infinitesimal range. Hamilton's principle is utilized for derivation of governing equations of motion for the shell. For thermal part of the problem, the two-dimensional coupled energy equation is assumed to govern the temperature field in the shell. Applying the Galerkin method in thickness direction of the shell, two-dimensional energy equation is reduced to two one-dimensional ones in meridian direction of the shell. To solve the fully coupled motion and energy equations, the finite element method is used for spatial discretization, and the Newmark method is used for temporal discretization. A validation case study is conducted by comparing the results of the present work and those which is obtained by the ABAQUS software.
\end{abstract}

\section{Keywords}

Dynamic coupled thermoelasticity, Thin spherical shell, Transverse thermal shock, Finite element analysis, Flexural element

Received: 23 April 2019; Accepted: 29 May 2019

ISSN: 2630-5763 (online) C 2019 Golden Light Publishing ${ }^{\circledR}$ All rights reserved.

\section{Introduction}

The coupled thermoelasticity refers to the mutual interaction of elastic and thermal deformations in solid bodies. Thus, the coupled thermoelastic analysis necessitates simultaneous solution of the associated motion and energy equations. Due to this nature, the governing equations of dynamic coupled thermoelasticity have complicated behavior and require a deep physical and mathematical understanding to solve them precisely.
One of the most important topics in thermoelasticity is the coupled thermoelastic analysis of structural members such as beams, plates, and shells. Structural members in various industries may be subjected to severe thermal loads. In such circumstances, the coupled thermoelastic analysis become necessary for evaluating dynamic thermo-mechanical fields through the structural members.

Up to now, many researches have been conducted on the coupled thermoelastic analysis of structural members. It seems that Ignaczak and

\footnotetext{
Corresponding author

E-mail: eslami@aut.ac.ir
} 
Nowacki [1] for the first time addressed such an analysis. They presented coupled thermoelastic formulations for dynamical analysis of plates under non-steady temperature distributions. Also, Jones [2] for the first time presented an investigation on the coupled thermoelastic behavior of beams. This work contains the Euler-Bernoulli and Timoshenko beam hypotheses, and presented formulations for beams with different thermal and mechanical boundary conditions. Also, researches on coupled thermoelasticity of beams have extended to geometrically nonlinear analysis [3], and beams made of functionally graded materials (FGMs) [4]. Among the early studies on the coupled thermoelastic analysis of thin plates, the work done by Inan [5] could be mentioned. This work implemented an analytical approach and investigated the free vibration as well as general solution of the associated motion equations of thin elastic plates for thermo-mechanically coupled conditions. Later, Chang and Wan [6] presented a Galerkin-based investigation on the thermomechanically coupled large amplitude vibrations of thin elastic plates. This work presents some results on damping aspect of coupled thermoelastic analysis, and a comparison between coupled and un-coupled results. Also, Trajkovski and Čukić [7] presented an inspiring work on the dynamic coupled thermoelastic behavior of circular plates under a surface thermal shock load. This work benefits a solution based on the integral transforms which has the capability for exact implementation of boundary conditions. For investigation on the effect of thermo-mechanical coupling on the nonlinear dynamics of orthotropic plates, Yeh [8] used a Galerkin-based solution method. This work presented for plates with simply supported boundary conditions, and plates' behavior was considered to be governed by the classical plate theory. The results of this work mainly show the effects of material and geometrical characteristics of plates, stemming from thermo-mechanical coupling and orthotropy of plates' material, on the amplitude decaying of their vibrational motion. Researches also was conducted to investigate the dynamic coupled thermoelastic behavior of plates made of FGMs. In this regard, works done by Akbarzadeh et al [9] and Jafarinezhad and Eslami [10], respectively, present dynamic thermoelastic analysis on behavior of rectangular and annular plates subjected to lateral thermal shocks. It seems that the leading work on the analysis of the dynamic thermoelastic response of shell structures belongs to McQuillen and Brull [11]. The numerical results of this work addressed the behavior of infinitelength cylindrical shells subjected to suddenly applied and rotating longitudinal lines of heat flux. Eslami et al. [12], by means of the Galerkin finite element procedure, investigated the dynamic thermoelastic response of long cylindrical shells subjected to thermal and mechanical shocks. Using the Ritz finite element method, Chang and Shyong [13] presented an interesting investigation on the vibrational behavior of laminated cylindrical shell panels subjected to a surface thermal shock. Through numerical results of this work, effects of thermo-mechanical coupling, boundary conditions, ply angles, and radius of curvature on dynamic thermoelastic response of laminated cylindrical shell panels were studied.

Eslami et al. [14] investigated the dynamic coupled thermoelastic behavior for shells of revolution based on the Flügge second-order shell theory. This work uses the Galerkin finite element method for solving the governing equations and exhibits the effect of normal stress and strain on the response of shells to thermal and mechanical shocks. The dynamic coupled thermoelastic response has also been studied for cylindrical shells made of FGMs, see the work by Bahtui and Eslami [15]. This work utilizes a second-order sheardeformable shell model as the kinematical idealization and a Galerkin trans-finite element method as the solution procedure.

It seems that the most of literature addressing the dynamic coupled thermoelastic analysis of shell structures deal with cylindrical ones and the works done on the spherical shells are rare. The work done by Kraus [16] is one of few works performed on the dynamic thermoelastic analysis of spherical shells. However, the work formulated in the context of uncoupled thermoelasticity theory. 
Consequently, the present work deals with the dynamic coupled thermoelastic response of spherical shells to suddenly applied thermal shocks. The work is formulated based on a first-order sheardeformable shell model. The shell is considered to obey Hooke's law for their mechanical material properties and to accept infinitesimal strains under the applied load conditions. The classical dynamic coupled energy equation is used for obtaining the temperature distribution through the shells. Also, caused by thinness assumption for shells, the temperature field in the shells thickness direction is approximated to vary linearly. Based on governing equations, a flexural coupled thermoelastic element is derived and its applicability is proved in comparison with ABAQUS software. Also, the effects of shells slenderness and thermal edge conditions on the dynamic coupled thermoelastic response of thin spherical shells to lateral thermal shocks are investigated.

\section{Governing equations}

\subsection{Equations of motion}

The distribution of displacement field is approximated as:

$$
\begin{aligned}
& U(\phi, \theta, z, t)=u_{0}(\phi, \theta, t)+z \psi_{\phi}(\phi, \theta, t) \\
& V(\phi, \theta, z, t)=v_{0}(\phi, \theta, t)+z \psi_{\theta}(\phi, \theta, t) \\
& W(\phi, \theta, z, t)=w_{0}(\phi, \theta, t)
\end{aligned}
$$

where $z$ expresses the thickness coordinate of shells such that the radial position for material points of the shell is expressed by $R+z$. Shells under applied loads are considered to have infinitesimal deformations [17,18]. Accordingly, in spherical coordinates, strain-displacement relations are obtained as (2).

$$
\begin{aligned}
& \varepsilon_{\phi \phi}=\frac{1}{R\left(1+\frac{z}{R}\right)}\left(\frac{\partial u_{0}}{\partial \phi}+w_{0}+z \frac{\partial \psi_{\phi}}{\partial \phi}\right) \\
& \varepsilon_{\phi z}=\frac{1}{\left(1+\frac{z}{R}\right)}\left(\frac{1}{R} \frac{\partial w_{0}}{\partial \phi}+\psi_{\phi}-\frac{u_{0}}{R}\right)
\end{aligned}
$$

$$
\begin{aligned}
\varepsilon_{\theta \theta}= & \frac{1}{R\left(1+\frac{z}{R}\right)}\left(\frac{1}{\sin \phi} \frac{\partial v_{0}}{\partial \theta}+\cot \phi u_{0}\right. \\
& \left.+w_{0}+\frac{z}{\sin \phi}\left(\cos \phi \psi_{\phi}\right)\right) \\
\varepsilon_{\theta z}= & 0, \quad \varepsilon_{\phi \theta}=0
\end{aligned}
$$

The material properties of shells are assumed to be linearly thermoelastic, so as the constitutive equations become

$$
\begin{aligned}
\sigma_{\phi \phi} & =\frac{E}{1-v^{2}}\left(\varepsilon_{\phi \phi}+v \varepsilon_{\theta \theta}\right)-\frac{E \alpha}{1-v} \mathfrak{T} \\
\sigma_{\theta \theta} & =\frac{E}{1-v^{2}}\left(\varepsilon_{\theta \theta}+v \varepsilon_{\phi \phi}\right)-\frac{E \alpha}{1-v} \mathfrak{T} \\
\sigma_{\theta z} & =\frac{E}{2(1+v)} \varepsilon_{\theta z} \\
\sigma_{\phi z} & =\frac{E}{2(1+v)} \varepsilon_{\phi z} \\
\sigma_{\phi \theta} & =\frac{E}{2(1+v)} \varepsilon_{\phi \theta}
\end{aligned}
$$

Using Hamilton's principle and variational calculus the motion equations for spherical shells become [17]:

$$
\begin{aligned}
& \frac{\partial}{\partial \phi}\left(R \sin \phi N_{\phi \phi}\right)-R \cos \phi N_{\theta \theta} \\
& \quad+R \sin \phi Q_{\phi}=\left(I_{0} \ddot{u}_{0}+I_{1} \ddot{\psi}_{\phi}\right) R^{2} \sin \phi \\
& \frac{\partial}{\partial \phi}\left(R \sin \phi Q_{\phi}\right)-R \sin \phi\left(N_{\phi \phi}+N_{\theta \theta}\right) \\
& \quad=I_{0} \ddot{w}_{0} R^{2} \sin \phi \\
& \frac{\partial}{\partial \phi}\left(R \sin \phi M_{\phi \phi}\right)-R \cos \phi M_{\theta \theta} \\
& \quad-R^{2} \sin \phi Q_{\phi}=\left(I_{1} \ddot{u}_{0}+I_{2} \ddot{\psi}_{\phi}\right) R^{2} \sin \phi
\end{aligned}
$$

where $I_{i} \mathrm{~s}$ are integral inertial constants, and are

$$
I_{i}=\int_{-\frac{h}{2}}^{\frac{h}{2}} \rho z^{i} d z
$$

Also, stress resultants appeared in (4) are defined as (6). 
$N_{i j}=\int_{-\frac{h}{2}}^{\frac{h}{2}} \sigma_{i j} d z, \quad i j=\phi \phi, \theta \theta, \phi \theta$

$M_{i j}=\int_{-\frac{h}{2}}^{\frac{h}{2}} \sigma_{i j} z d z, \quad i j=\phi \phi, \theta \theta$

$Q_{i}=\int_{-\frac{h}{2}}^{\frac{h}{2}} \sigma_{i z} d z, \quad i=\phi, \theta$

Along with motion equations, the associated boundary conditions for spherical shells are obtained. These boundary conditions could be presented as (7).

$$
\begin{array}{llll}
\delta u_{0}=0 & \text { or } & N_{\phi \phi}=\bar{N}_{\phi \phi} \\
\delta \psi_{\phi}=0 & \text { or } & M_{\phi \phi}=\bar{M}_{\phi \phi} \\
\delta w_{0}=0 & \text { or } & Q_{\phi}=\bar{Q}_{\phi}
\end{array}
$$

\subsection{Energy equations}

In Cartesian coordinate system, the energy equation associated with linear dynamic coupled thermoelasticity using indicial notation and summation convention is as (8) [18]. For more information on the linear dynamic coupled thermoelasticity see $[19,20]$.

$$
\rho C_{\epsilon} \dot{\mathfrak{T}}+T_{0} \beta_{i j} \varepsilon_{i j}=\left(k_{i j} \mathfrak{T}_{j}\right)_{, i}
$$

where $i, j=1,2,3$. This equation in spherical coordinates and for axisymmetric analysis become:

$$
\begin{array}{r}
k\left(\frac{1}{R^{2}} \frac{\partial}{\partial z}\left(R^{2} \frac{\partial \mathfrak{T}}{\partial z}\right)+\frac{1}{R^{2}} \frac{1}{\sin \phi} \frac{\partial}{\partial \phi}\left(\sin \phi \frac{\partial \mathfrak{T}}{\partial \phi}\right)\right) \\
-\rho C_{\epsilon} \dot{\mathfrak{T}}-\beta T_{0}\left(\dot{\varepsilon}_{z z}+\dot{\varepsilon}_{\phi \phi}+\dot{\varepsilon}_{\theta \theta}\right)=0
\end{array}
$$

where $\beta=(3 \lambda+2 \mu) \alpha$. In this work, it is assumed that the temperature varies linearly through the shell thickness. So, the mathematical statement of temperature distribution through the shell is as (10).

$$
\mathfrak{T}(\phi, \theta, z, t)=\mathfrak{T}_{0}(\phi, \theta, t)+z \mathfrak{T}_{1}(\phi, \theta, t)
$$

For imposing the prescribed temperature distribution on the energy equation, Galerkin method is utilized. In this way, the coefficients of Eq. (10) are made orthogonal to (9) in thickness direction of shells. Implementing the mentioned process leads to two reduced energy equations, i.e. Eq. (11), that determine distribution of $\mathfrak{T}_{0}$ and $\mathfrak{T}_{1}$ through the spherical shells.

$$
\begin{gathered}
\rho C_{\epsilon} h \dot{\mathfrak{T}}_{0}+\beta T_{0} \frac{h}{R}\left(\dot{u}_{0}+2 \dot{w}_{0}+\dot{u}_{0} \cot \phi\right) \\
-q_{z}-\frac{k h}{R^{2} \sin \phi} \frac{\partial}{\partial \phi}\left(\sin \phi \frac{\partial \mathfrak{T}_{0}}{\partial \phi}\right)=0 \\
\rho C_{\epsilon} \frac{h^{3}}{12} \dot{\mathfrak{T}}_{1}+\beta T_{0} \frac{h^{3}}{12 R} \dot{\psi}_{\phi}(1+\cot \phi)-q_{z} \frac{h}{2} \\
+k h \mathfrak{T}_{1}-\frac{k h^{3}}{12 R^{2} \sin \phi} \frac{\partial}{\partial \phi}\left(\sin \phi \frac{\partial \mathfrak{T}_{1}}{\partial \phi}\right)=0
\end{gathered}
$$

\subsection{Weak formulation}

Derivation of weak-form for governing equations is rooted in the concept of weighted residual integrals. The process begins with the orthogonalization of motion and energy equations to the test functions, i.e. $\delta u_{0}, \delta w_{0}, \delta \psi_{\phi}, \delta \mathfrak{T}_{0}$, and $\delta \mathfrak{T}_{1}$. In this section, $\mathcal{S}$ indicates the curved-path along meridional direction of shells, and located at their mid-surface. For spherical shells $\mathcal{S}=R \phi$ and $d \mathcal{S}=R d \phi$. The weighted integral-form associated with governing equations of spherical shells may be written as Eq. (12).

$$
\begin{array}{r}
\int_{\mathcal{S}} \delta u_{0}\left(\frac{\partial}{\partial \phi}\left(R \sin \phi N_{\phi \phi}\right)-R \cos \phi N_{\theta \theta}+R \sin \phi Q_{\phi}\right. \\
\left.-\left(I_{0} \ddot{u}_{0}+I_{1} \ddot{\psi}_{\phi}\right) R^{2} \sin \phi\right) d \mathcal{S}=0 \\
(12 \mathrm{a}) \\
\int_{\mathcal{S}} \delta w_{0}\left(\frac{\partial}{\partial \phi}\left(R \sin \phi M_{\phi \phi}\right)-R \cos \phi M_{\theta \theta}\right. \\
\left.-R^{2} \sin \phi Q_{\phi}-\left(I_{1} \ddot{u}_{0}+I_{2} \ddot{\psi}_{\phi}\right) R^{2} \sin \phi\right) d \mathcal{S}=0
\end{array}
$$

$$
\begin{array}{r}
\int_{\mathcal{S}} \delta \psi_{\phi}\left(\frac{\partial}{\partial \phi}\left(R \sin \phi Q_{\phi}\right)-R \sin \phi\left(N_{\phi \phi}+N_{\theta \theta}\right)\right. \\
\left.-I_{0} \ddot{w}_{0} R^{2} \sin \phi\right) d \mathcal{S}=0
\end{array}
$$




$$
\begin{array}{r}
\int_{\mathcal{S}} \delta \mathfrak{T}_{0}\left(\rho C_{\epsilon} h \dot{\mathfrak{T}}_{0}+\beta T_{0} \frac{h}{R}\left(\dot{u}_{0}+2 \dot{w}_{0}+\dot{u}_{0} \cot \phi\right)\right. \\
\left.-q_{z}-\frac{k h}{R^{2} \sin \phi} \frac{\partial}{\partial \phi}\left(\sin \phi \frac{\partial \mathfrak{T}_{0}}{\partial \phi}\right)\right) d \mathcal{S}=0
\end{array}
$$

$$
\begin{array}{r}
\int_{\mathcal{S}} \delta \mathfrak{T}_{1}\left(\rho C_{\epsilon} \frac{h^{3}}{12} \dot{\mathfrak{T}}_{1}+\beta T_{0} \frac{h^{3}}{12 R} \dot{\psi}_{\phi}(1+\cot \phi)-q_{z} \frac{h}{2}\right. \\
\left.+k h \mathfrak{T}_{1}-\frac{k h^{3}}{12 R^{2} \sin \phi} \frac{\partial}{\partial \phi}\left(\sin \phi \frac{\partial \mathfrak{T}_{1}}{\partial \phi}\right)\right) d \mathcal{S}=0
\end{array}
$$

Integrating Eqs. (12) by part and using Gauss's theorem in conjunction with some variational principles, the weak form of governing equations is obtained [21].

\section{Solution procedure}

\subsection{Spatial discretization}

In this work, the weak-form of governing equations are spatially discretized using standard displacement-based finite element procedure. For this purpose, filed variables, namely $u_{0}, w_{0}, \psi_{\phi}$, $\mathfrak{T}_{0}$, and $\mathfrak{T}_{1}$, are discretized as Eqs. (13).

$$
\begin{aligned}
& u_{0}(\mathcal{S}, t) \approx \mathbf{N}^{u_{0}}(\mathcal{S}) \cdot \mathbf{X}^{u_{0}}(t) \\
& w_{0}(\mathcal{S}, t) \approx \mathbf{N}^{w_{0}}(\mathcal{S}) \cdot \mathbf{X}^{w_{0}}(t) \\
& \psi_{\phi}(\mathcal{S}, t) \approx \mathbf{N}^{\psi \phi}(\mathcal{S}) \cdot \mathbf{X}^{\psi \phi}(t) \\
& \mathfrak{T}_{0}(\mathcal{S}, t) \approx \mathbf{N}^{\mathfrak{T}_{0}}(\mathcal{S}) \cdot \mathbf{X}^{\mathfrak{T}_{0}}(t) \\
& \mathfrak{T}_{1}(\mathcal{S}, t) \approx \mathbf{N}^{\mathfrak{T}_{1}}(\mathcal{S}) \cdot \mathbf{X}^{\mathfrak{T}_{1}}(t)
\end{aligned}
$$

Substituting Eqs. (13) into weak-form of governing equations along with some mathematical operations leads to associated finite element equations, and may be arranged as following matrix form

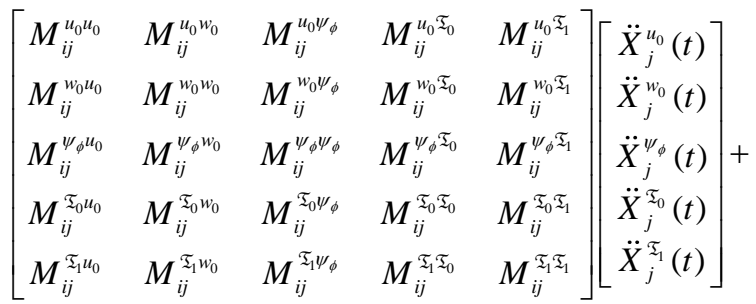

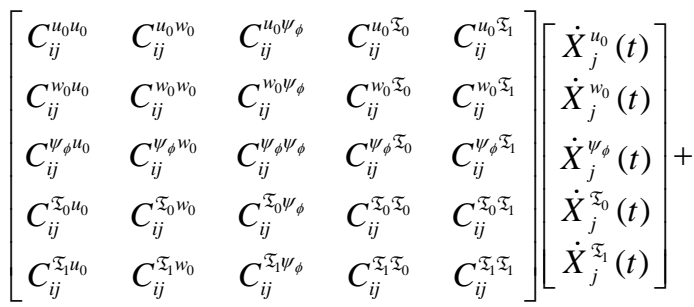

$$
\begin{aligned}
& {\left[\begin{array}{lllll}
K_{i j}^{u_{0} u_{0}} & K_{i j}^{u_{0} w_{0}} & K_{i j}^{u_{0} \psi_{\phi}} & K_{i j}^{u_{0} \mathfrak{T}_{0}} & K_{i j}^{u_{0} \mathfrak{T}_{1}} \\
K_{i j}^{w_{0} u_{0}} & K_{i j}^{w_{0} w_{0}} & K_{i j}^{w_{0} \psi_{\phi}} & K_{i j}^{w_{0} \mathfrak{T}_{0}} & K_{i j}^{w_{0} \mathfrak{T}_{1}} \\
K_{i j}^{\psi_{\phi} u_{0}} & K_{i j}^{\psi_{\phi} w_{0}} & K_{i j}^{\psi_{\phi} \psi_{\phi}} & K_{i j}^{\psi_{\phi} \mathfrak{T}_{0}} & K_{i j}^{\psi_{\phi} \mathfrak{I}_{1}} \\
K_{i j}^{\mathfrak{T}_{0} u_{0}} & K_{i j}^{\mathfrak{T}_{0} w_{0}} & K_{i j}^{\mathfrak{T}_{0} \psi_{\phi}} & K_{i j}^{\mathfrak{T}_{0} \mathfrak{T}_{0}} & K_{i j}^{\mathfrak{T}_{0} \mathfrak{\tau}_{1}} \\
K_{i j}^{\mathfrak{T}_{1} u_{0}} & K_{i j}^{\mathfrak{T}_{1} w_{0}} & K_{i j}^{\mathfrak{T}_{1} \psi_{\phi}} & K_{i j}^{\mathfrak{T}_{1} \mathfrak{T}_{0}} & K_{i j}^{\mathfrak{T}_{1} \mathfrak{q}_{1}}
\end{array}\right]\left[\begin{array}{c}
X_{j}^{u_{0}}(t) \\
X_{j}^{w_{0}}(t) \\
X_{j}^{\psi_{\phi}}(t) \\
X_{j}^{\mathfrak{T}_{0}}(t) \\
X_{j}^{\mathfrak{T}_{1}}(t)
\end{array}\right]=\left[\begin{array}{c}
F_{j}^{u_{0}}(t) \\
F_{j}^{w_{0}}(t) \\
F_{j}^{\psi_{\phi}}(t) \\
F_{j}^{\mathfrak{T}_{0}}(t) \\
F_{j}^{\mathfrak{T}_{1}}(t)
\end{array}\right]}
\end{aligned}
$$




\subsection{Temporal discretization}

The Newmark method is adjusted for temporal discretization of Eq. (14). This is a dynamic updating procedure which converts the differentialalgebraic equations to the algebraic system of equations. Assume that the global finite element equations obtained in the previous section could be shown by Eq. (15).

$$
\mathcal{M} \ddot{\mathbf{X}}+\mathcal{C} \dot{\mathbf{X}}+\mathcal{K} \mathbf{X}+\mathbf{f}=0
$$

Based on Newmark's method, the unknown vector and its time derivatives at the time step $n+1$ are approximated according to Eq. (16)

$$
\begin{aligned}
& \mathbf{X}_{n+1}=\mathbf{X}_{n}+\Delta t \dot{\mathbf{X}}_{n}+\frac{1}{2}(\Delta t)^{2} \ddot{\mathbf{X}}_{n+\gamma} \\
& \dot{\mathbf{X}}_{n+1}=\dot{\mathbf{X}}_{n}+\Delta t \ddot{\mathbf{X}}_{n+\varphi} \\
& \ddot{\mathbf{X}}_{n+\varphi}=(1-\varphi) \ddot{\mathbf{X}}_{n}+\varphi \ddot{\mathbf{X}}_{n+1}
\end{aligned}
$$

where $\gamma$ and $\varphi$ are the parameters that determine the explicit/implicit form of the solution procedure. Substituting Eqs. (16) into Eq. (15) leads to the following system of algebraic equations

$$
\check{\mathcal{K}}_{n+1} \mathbf{X}_{n+1}+\dot{\mathbf{f}}_{n, n+1}=0
$$

where

$$
\begin{aligned}
& \dot{\mathcal{K}}_{n+1}=\mathcal{K}_{n+1}+a_{3} \mathcal{M}_{n+1}+a_{6} \mathcal{C}_{n+1} \\
& \mathbf{f}_{n, n+1}=\mathbf{f}_{n+1}+\mathcal{M}_{n+1} \mathbf{y}_{n}^{M}+\mathcal{C}_{n+1} \mathbf{y}_{n}^{C} \\
& \mathbf{y}_{n}^{M}=a_{3} \mathbf{X}_{n}+a_{4} \dot{\mathbf{X}}_{n}+a_{5} \ddot{\mathbf{X}}_{n} \\
& \mathbf{y}_{n}^{C}=a_{6} \mathbf{X}_{n}+a_{7} \dot{\mathbf{X}}_{n}+a_{8} \ddot{\mathbf{X}}_{n} \\
& a_{3}=\frac{2}{\gamma(\Delta t)^{2}}, \quad a_{4}=\Delta t a_{3}, \\
& a_{5}=\frac{1}{\gamma}-1, \quad a_{6}=\frac{2 \varphi}{\gamma \Delta t}, \\
& a_{7}=\frac{2 \varphi}{\gamma}-1, \quad a_{8}=\Delta t\left(\frac{\varphi}{\gamma}-1\right)
\end{aligned}
$$

For first time step, knowledge of $\mathbf{X}_{0}, \mathbf{X}_{0}$, and $\ddot{X}_{0}$ are required. The values of $\mathbf{X}_{0}$ and $\dot{\mathbf{X}}_{0}$ are provided by physical initial conditions and the value of $\ddot{\mathbf{X}}_{0}$ is obtained using Eq. (15). Also, at the end of each time step the new first and second time derivatives of unknowns are computed as

$$
\begin{aligned}
& \ddot{\mathbf{X}}_{n+1}=a_{3}\left(\mathbf{X}_{n+1}-\mathbf{X}_{n}\right)-a_{4} \dot{\mathbf{X}}_{n}-a_{5} \ddot{\mathbf{X}}_{n} \\
& \dot{\mathbf{X}}_{n+1}=\dot{\mathbf{X}}_{n}+a_{2} \ddot{\mathbf{X}}_{n}+a_{1} \ddot{\mathbf{X}}_{n+1}
\end{aligned}
$$

where $a_{1}=\varphi \Delta t$ and $a_{2}=(1-\varphi) \Delta t$.

\section{Results and discussions}

The finite element model presented in this work can be used for numerical investigation on the response of thin spherical shells under axisymmetric loading conditions. In this section, a spherical shell of revolution which occupies the region between $\phi=30^{\circ}$ and $\phi=60^{\circ}$ is considered. The thermomechanical material properties of the shell are presented in Table 1. Shells initially are considered to be at $293 \mathrm{~K}$ temperature. Then, the outer surface of the shells is considered to be subjected by a steptype heat flux loading that its magnitude is $10^{4} \mathrm{~W} / \mathrm{m}^{2}$. It should be noted that the inner surface of shells is considered to be thermally insulated, and the boundary edges of the shells mechanically are assumed to be clamped.

Table 1. Thermo-mechanical properties of spherical shells

\begin{tabular}{ll}
\hline Parameter & Value \\
\hline$\rho$ & $7904 \mathrm{~kg} / \mathrm{m}^{3}$ \\
$E$ & $200 \mathrm{GPa}$ \\
$v$ & 0.3 \\
$k$ & $50 \mathrm{~W} / \mathrm{mK}$ \\
$\alpha$ & $11.8 \times 10^{-6} \quad 1 / \mathrm{K}$ \\
$C_{\epsilon}$ & $500 \mathrm{~J} / \mathrm{kgK}$ \\
\hline
\end{tabular}




\subsection{Validation study}

For the purpose of validation, the performance of developed flexural element is compared with that of ABAQUS C3D8T element. The shells under investigation are considered to have the geometrical slenderness of $\mathrm{R} / \mathrm{h}=50$. Also, the boundary edges of the shells during the analysis are assumed to be kept at the initial temperature. Under the applied thermal shock, response of the shell is illustrated in Fig. 1. In this figure, the response of considered spherical shell has been obtained both with flexural element developed in this work, and the C3D8T element of ABAQUS software. As seen, Figs. 1a and $1 \mathrm{~b}$ show that for both lateral deflection and temperature rise the results of two elements are in good agreement with each other. This agreement is seen for non-steady as well as steady parts of solutions. Also, the validation study has been performed for distribution of temperature rise through the thickness of shells, Fig. 1c. The maximum difference seen between results of two elements is about 2 percent. This means that the linear temperature approximation in the thickness direction is a reasonable choice for dynamic coupled thermoelastic analysis of thin spherical shells. In this way, the performance of the developed flexural element for coupled dynamic thermoelastic analysis of thin spherical shells is confirmed.

\subsection{Parametric study}

In this section, the effects of slenderness parameter and thermal conditions at boundary edges on the response of thin spherical shells are studied. Consider Figs. 2 and 3, figures reveal that the greater slenderness ratio for a shell results in greater temperature rise in a certain time period. Because of thermally insulated inner surface of the shell, the greater temperature rise is followed by larger lateral deflection for both types of thermal boundary edges. Also, comparing Figs. 2 and 3 reveals that the insulating the boundary edges of the shell results in greater temperature elevation through the shell at any arbitrary frame of time.

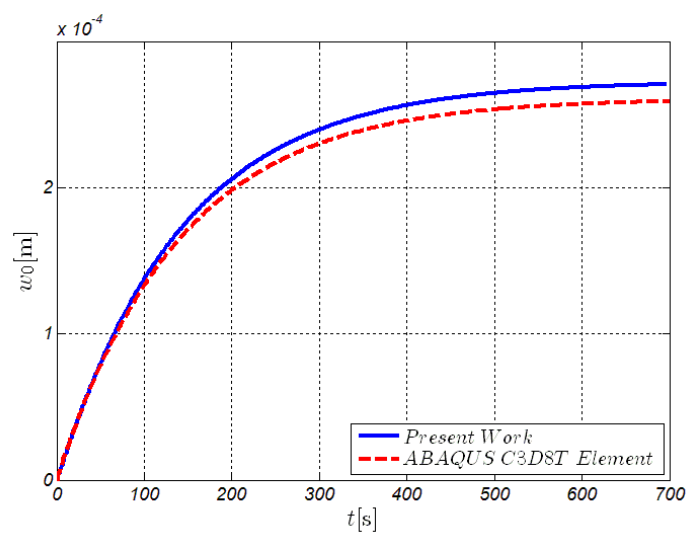

(a)

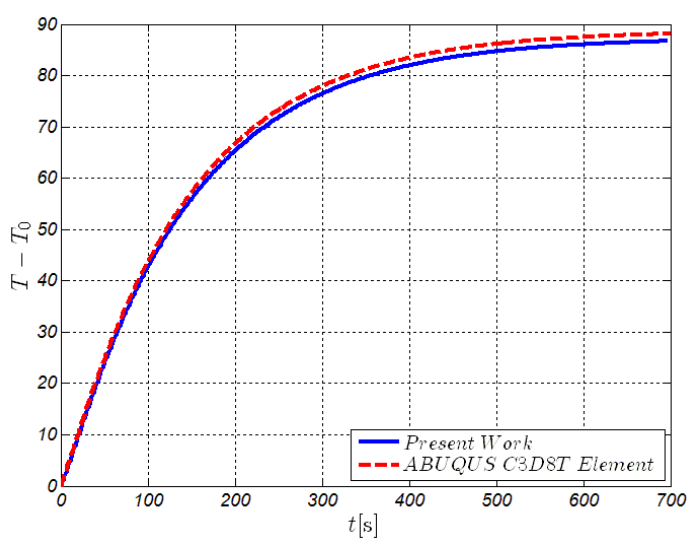

(b)

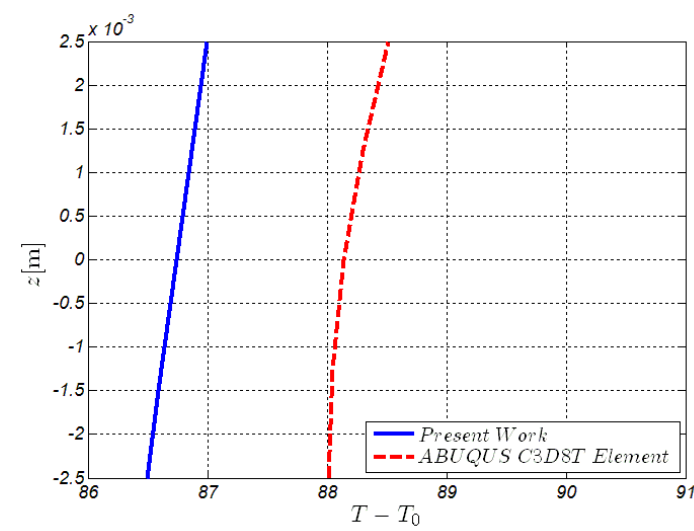

(c)

Fig. 1. Comparison between the results of flexural element developed in this work and C3D8T element of ABAQUS software: (a) Lateral deflection of point $\phi=45^{\circ}$ with respect to time. (b) Temperature of point $z=0, \phi=45^{\circ}$ with respect to time. (c) Distribution of temperature through the thickness of shell at point $\phi=45^{\circ}$ 
In addition, caused by heat transfer at isothermal boundary edges, both lateral deflection and temperature rise of the shells reach a steady-state condition. However, these steady states do not appear in the results belong to thermally insulated edges.

\section{Conclusions}

Present work addressed the dynamic coupled thermoelastic response of thin spherical shells to suddenly applied lateral thermal shocks. The work has been formulated based on a first-order sheardeformable shell model. The shell has been considered to obey Hooke's law for their mechanical material properties and to accept infinitesimal strains under the applied load conditions. The classical dynamic coupled energy equation has been used for obtaining the temperature distribution through the shells. Also, the linear approximation function has been considered to govern temperature distribution in thickness direction of shells. Based on governing equations, a flexural coupled thermoelastic element has been derived and its applicability has been confirmed in comparison with ABAQUS C3D8T element. Results reveal the effects of shells slenderness and thermal edge conditions on the dynamic coupled thermoelastic response of thin spherical shells to lateral thermal shocks. Accordingly, some points could be highlighted as concluding remarks:

- Developed flexural element could be a reliable alternative for 3D coupled thermoelastic elements.

- The linear approximation seems to be good enough for temperature evaluation in thickness direction of shells.

- The slenderer shells experience the more temperature rise and consequently the more lateral deflection under the applied thermal shock and boundary conditions.

- Although the isothermal edge condition results in the steady-state temperature rise and lateral deflection, these steady states do not happen for shells with thermally insulated edge conditions.

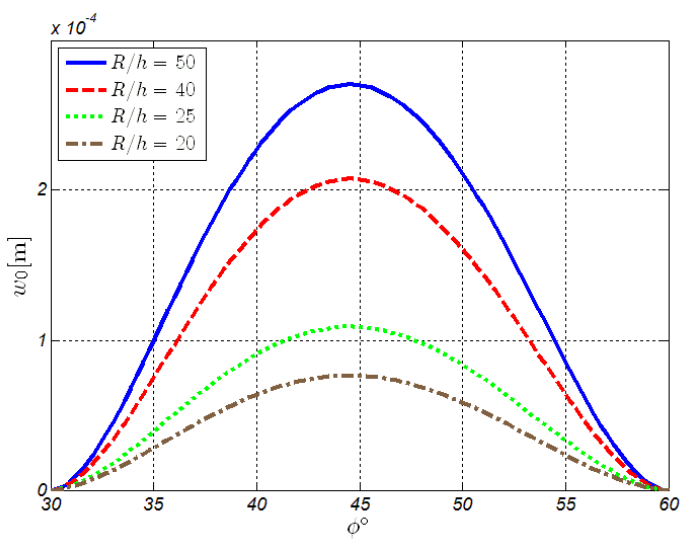

(a)

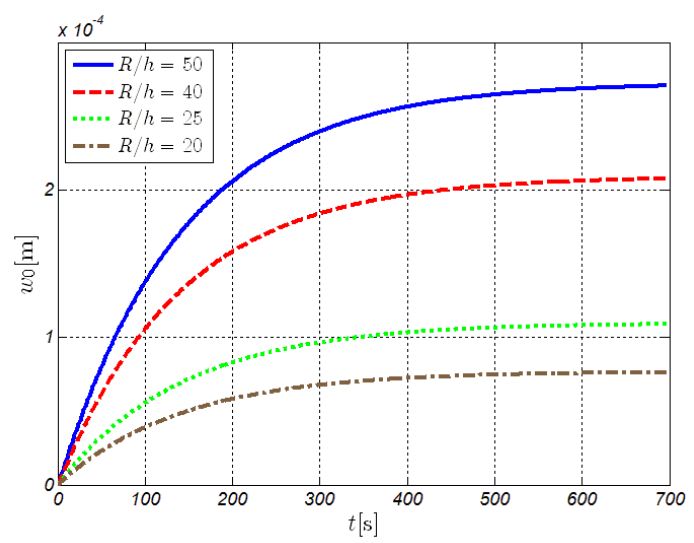

(b)

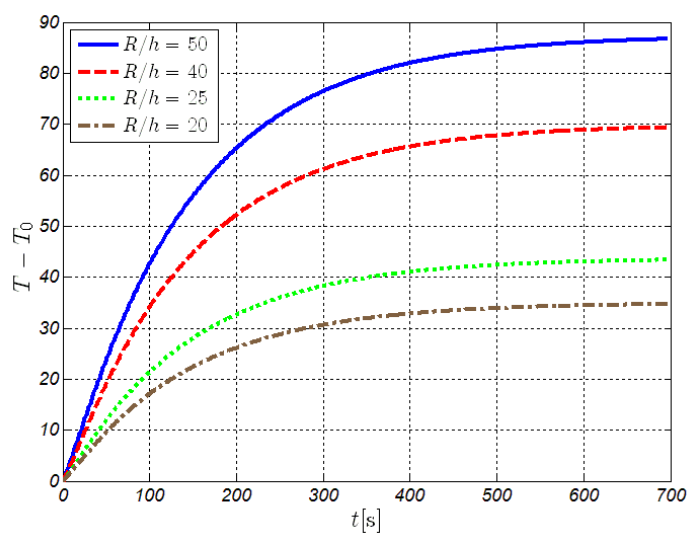

(c)

Fig. 2. Effect of slenderness on the response of thin spherical shells with isothermal boundary edges: (a) Lateral deflection through the meridian direction of shells. (b) Lateral deflection of point $\phi=45^{\circ}$ with respect to time. (c) Temperature of point $z=0, \phi=45^{\circ}$ with respect to time 


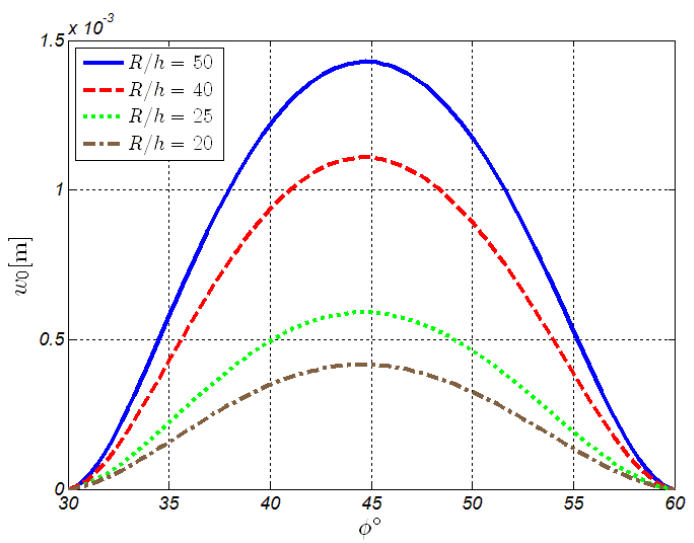

(a)

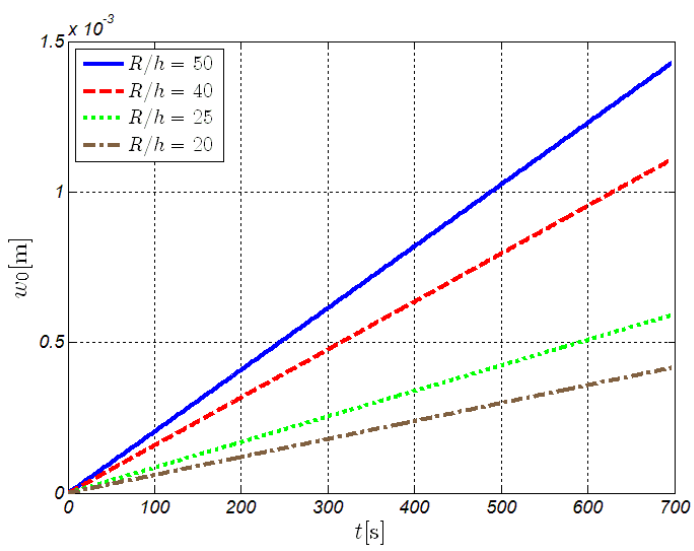

(b)

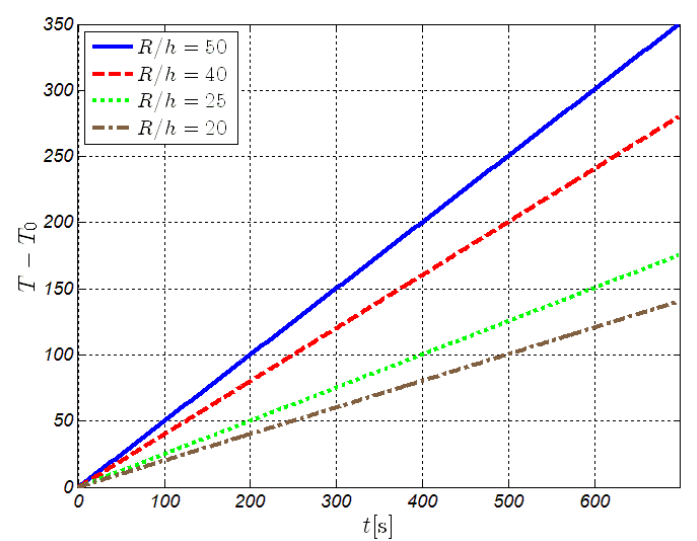

(c)

Fig. 3. Effect of slenderness on the response of thin spherical shells with thermally insulated boundary edges: (a) Lateral deflection through the meridian direction of shells. (b) Lateral deflection of point $\phi=45^{\circ}$ with respect to time. (c) Temperature of point $z=0, \phi=45^{\circ}$ with respect to time.

\section{References}

[1] Ignaczak J, Nowacki W (1961) Transversal vibration of a plate, produced by heating. Archiwum Mechaniki Stosowanej 650-770.

[2] Jones JP (1966) Thermoelastic vibrations of a beam. The Journal of the Acoustical Society of America 39(3): 542-548.

[3] Manoach E, Ribeiro P (2004) Coupled, thermoelastic, large amplitude vibrations of Timoshenko beams. International Journal of Mechanical Sciences 46(11): 1589-1606.

[4] Babaei MH, Abbasi M, Eslami MR (2008) Coupled thermoelasticity of functionally graded beams. Journal of Thermal Stresses 31(8): 680697.

[5] Inan E. (1972) Coupled theory of thermoelastic plates. Acta Mechanica 14(1): 1-29.

[6] Chang WP, Wan SM (1986) Thermomechanically coupled non-linear vibration of plates. International Journal of Non-Linear Mechanics 21(5): 375-389.

[7] Trajkovski D, Čukić R (1999) A coupled problem of thermoelastic vibrations of a circular plate with exact boundary conditions. Mechanics Research Communications 26(2): 217-224.

[8] Yeh YL (2005) The effect of thermo-mechanical coupling for a simply supported orthotropic rectangular plate on non-linear dynamics. Thinwalled structures 43(8): 1277-1295.

[9] Akbarzadeh AH, Abbasi M, Eslami MR (2012) Coupled thermoelasticity of functionally graded plates based on the third-order shear deformation theory. Thin-Walled Structures 53: 141-155.

[10] Jafarinezhad MR, Eslami MR (2017) Coupled thermoelasticity of FGM annular plate under lateral thermal shock. Composite Structures 168: 758771.

[11] McQuillen EJ, Brull MA (1970) Dynamic thermoelastic response of cylindrical shells. Journal of Applied Mechanics 37(3): 661-670.

[12] Eslami MR, Shakeri M, Sedaghati R (1994) Coupled thermoelasticity of an axially symmetric cylindrical shell. Journal of Thermal Stresses 17(1): 115-135.

[13] Chang JS, Shyong JW (1994) Thermally induced vibration of laminated circular cylindrical shell panels. Composites Science and Technology 51(3): 419-427.

[14] Eslami MR, Shakeri M, Ohadi AR, Shiari B (1999) Coupled thermoelasticity of shells of revolution: 
effect of normal stress and coupling. AIAA Journal 37(4): 496-504.

[15] Bahtui A, Eslami MR (2007) Coupled thermoelasticity of functionally graded cylindrical shells. Mechanics Research Communications. 34(1): 1-18.

[16] Kraus H (1966) Thermally induced vibrations of thin nonshallow spherical shells. AIAA Journal, 4(3): 500-505.

[17] Reddy JN. Theory and Analysis of Elastic Plates and Shells. CRC Press, 2006.

[18] Hetnarski RB, Eslami MR. Thermal Stresses: Advanced Theory and Applications. Springer, Switzerland, 2019.

[19] Bateni M, Eslami MR (2017) Thermally nonlinear generalized thermoelasticity of a layer. Journal of Thermal Stresses 40(10): 1320-1338.

[20] Bateni M, Eslami MR (2018) Thermally nonlinear generalized thermoelasticity: A note on the thermal boundary conditions. Acta Mechanica 229(2): 807826.

[21] Eslami MR. Finite Elements Methods in Mechanics. Springer, 2014.

\section{Nomenclature}

$(\cdot),_{p} \quad$ Partial derivative with respect to $p$

$\beta \quad$ Thermoelastic coupling constant for isotropic materials

$\beta_{i j} \quad$ Components of Thermoelastic coupling tensor

Inner product symbol

$\delta \quad$ Variational operator

$\lambda \quad$ Normal Lamé constant

$\mu \quad$ Shear Lamé constant

$v \quad$ Poision's ratio

$\psi_{\phi} \quad$ Meridional cross-section rotation of spherical shells

$\psi_{\theta} \quad$ Circumferential cross-section rotation of spherical shells

$\rho \quad$ Mass density

$\sigma_{i j} \quad$ Components of Cauchy stress tensor

$\varepsilon_{i j} \quad$ Components of infinitesimal strain tensor

$C_{\epsilon} \quad$ Specific heat

E Elasticity modulus $h \quad$ Thickness of spherical shells

$R \quad$ Radius of curvature for mid-surface of spherical shells

$U \quad$ Meridional displacement for a material point of spherical shells

$u_{0} \quad$ Meridional displacement for mid-surface of spherical shells

$V \quad$ Circumferential displacement for a material point of spherical shells

$v_{0} \quad$ Circumferential displacement for mid-surface of spherical shells

W Radial displacement for a material point of spherical shells

$w_{0} \quad$ Radial displacement for mid-surface of spherical shells

$\mathfrak{T}$ Temperature difference with respect to stressfree configuration

$k \quad$ Thermal conductivity coefficient

$k_{i j} \quad$ Components of Thermal conductivity tensor

$T_{0} \quad$ Temperature of shells at stress-free configuration

$T \quad$ Absolute temperature

$\alpha \quad$ Coefficient of volumetric thermal expansion

$\mathbf{N}^{p} \quad$ Vector of shape functions associated with the variable $\mathrm{p}$

X Global vector of nodal unknowns

$\mathbf{X}$ Position vector of the material points

$\mathbf{X}^{p} \quad$ Vector of nodal unknowns associated with the variable $\mathrm{p}$

$q_{z} \quad$ Heat flux on the outer surface of shells 